\title{
Incoherent dynamics of vibrating single-molecule transistors.
}

\author{
Kevin D. McCarthy, Nikolay Prokof'ev, and Mark T. Tuominen \\ Department of Physics, University of Massachusetts, Amherst, MA 01003, USA
}

(Dated: October 30, 2018)

\begin{abstract}
We study the tunneling conductance of nano-scale quantum "shuttles" in connection with a recent experiment [H. Park et al., Nature, 407, 57 (2000)] in which a vibrating $\mathrm{C}_{60}$ molecule was apparently functioning as the island of a single electron transistor (SET). While our calculation starts from the same model of previous work [D. Boese and H. Schoeller, Europhys. Lett. 54, 668 (2001)] we obtain quantitatively different dynamics. Calculated I-V curves exhibit most features present in experimental data with a physically reasonable parameter set, and point to a strong dependence of the oscillator's potential on the electrostatics of the island region. We propose that in a regime where the electric field due to the bias voltage itself affects island position, a "catastrophic" negative differential conductance (NDC) may be realized. This effect is directly attributable to the magnitude of overlap of final and initial quantum oscillator states, and as such represents experimental control over quantum transitions of the oscillator via the macroscopically controllable bias voltage.
\end{abstract}

PACS numbers: 73.63-b,73.23Hk,72.20Dp

\section{INTRODUCTION}

Over the past decade, nanotechnology has advanced the ability to build systems in which chemical selfassembly defines the functional and structural units of nanoelectronic devices [4, 5, 6, 7]. Since elastic parameters of organic compounds currently utilized in such work can be much "softer" than those of semiconductors or metals, one must carefully consider the important role mechanical degrees of freedom may play in charge transport devices 8]. It is also fundamentally interesting to consider how the physics develops as one proceeds from the nanoscale all the way up to macroscopic electromechanical systems [9, 10].

A significant impetus in the field of chemically self assembled nanostructures is in the direction of Coulomb blockade devices, in which transport is due to the tunneling of a single electron or Cooper pair through the device via island structures of small capacitance $5,11,12,13$. The quest for systems with ever smaller capacitance has driven workers to define smaller structures in such devices, with the result that current experimental work, such as that modeled here, involves the utilization of single large molecules as the island structure through which electronic transport takes place.

The experiment of Ref. 1 involves the fabrication of metallic gaps roughly $1 \mathrm{~nm}$ in separation [14], and exposure to a solution of $C_{60}$ molecules. When IV curves were measured, some of the gaps exposed to $C_{60}$ displayed a robust $100 \mathrm{meV}$ Coulomb blockade with charging energy modulation via a third gate terminal (in this case the gate terminal was the underlying semiconductor substrate). Also observed was a series of smaller 5 $\mathrm{meV}$-wide steps attributable to coupling of the electronic transport through the island to the quantized vibrations of the center of mass motion of the molecule in its Van der Waals binding potential nearest to one of the leads. The steps appear to have some remarkable secondary features, i.e., asymmetries about zero bias voltage, and unresolved structure in the intra-step regions of the IV characteristics.

It was suggested in Ref. 1 that the number of vibrational steps and their amplitudes are controlled by Franck-Condon physics. This idea was further developed in Ref. 3 where Franck-Condon matrix elements were used to calculate transition rates between different vibrational states and to solve the master equation for the I-V curves. Our work differs from that of Ref. 3 in the following respects: i.) While we work with the same model as Ref. 3, we arrive at a different functional form for the transition matrix elements, with the result that our current voltage characteristics disagree quantitatively. ii.) Because of the unitary nature of the space-shift operators that must act on the electron tunneling perturbation, we stress the distinction of shuttle transport into unitary and non-unitary limits, depending on the degree to which energy conservation allows the tunneling perturbation can fully connect the Hilbert spaces of states before and after the island is charged. iii.) We also argue that a bias voltage dependence of the Franck-Condon factor should be considered, and that in fact such a dependence may explain the aforementioned secondary features in the IV characteristics of Ref. 1. A stronger dependence is further shown to lead to a "catastrophic" negative differential conductance effect which we will describe in this paper. The Chalmers group has modelled the system with classical, damped oscillations and incoherent electronics [8], and with classical undamped oscillations and electronic coherence 2]. Our model is that of incoherent electronics and quantum mechanical oscillations that are strongly damped. As compared with the model of Ref. 8 , the molecular island displays its vibrational character not by shuttling charge at a rate $\omega_{o}$, but in a more "spectroscopic" manner, as a sort of fingerprint in the charge transport physics. Strong dissipation of the oscillator's 
motion plays a key role in our incoherent dynamics, in contrast to 2], which assumes undamped classical motion.

\section{THEORETICAL MODEL}

Fig. (11) shows a depiction of the $C_{60}$ transistor system. The physics is assumed to be that of a 3 terminal quantum dot (including a capacitively coupled gate terminal) vibrating in a 1-d quantum harmonic oscillator potential (we disregard vibrational states in other directions by assuming that their frequencies are much higher). We write the system Hamiltonian as follows:

$$
\begin{aligned}
\hat{H}=\hat{H}_{i}+\sum_{s= \pm 1} \hat{H}_{s}+\hat{H}_{\mathbf{T}} & +\frac{e^{2}\left(\hat{n}-n_{o}\right)^{2}}{2 C}-\hat{n} E \hat{x} \\
& +\hat{V}_{\text {int }}^{\text {(bath) }}+\hat{H}_{\text {Bath }} .
\end{aligned}
$$

The vibrational portion of the island Hamiltonian is that of a simple harmonic oscillator in terms of the island center of mass momentum $p$ and coordinate $x$

$$
\hat{H}_{i}=\frac{\hat{p}^{2}}{2 m}+\frac{m \omega_{o}^{2} \hat{x}^{2}}{2} \equiv \hbar \omega_{o}\left(a^{\dagger} a+\frac{1}{2}\right) .
$$

The right $(s=1)$ and left $(s=-1)$ metallic leads are described by $\hat{H}_{s}$ as normal Fermi liquids with constant Fermi-surface density of states $\rho_{F}$, and chemical potentials controlled by the bias voltage. Charge transport between the island and leads is treated within the tunneling Hamiltonian approach

$$
\hat{H}_{\mathbf{T}}=t \sum_{s, k, \sigma}\left(c_{s k \sigma}^{\dagger} c_{i \sigma} e^{s x / \lambda}+h . c .\right)
$$

where $c_{s k \sigma}^{\dagger}$ and $c_{i \sigma}^{\dagger}$ are the electron creation operators in the leads and island respectively. Although spin is conserved during all tunneling events we need to keep track of island electronic spin states, since tunneling events in the two spin channels are correlated. Here $t$ is a constant, position independent tunneling matrix element, with the island centered between the metallic leads. Since the island is free to move, we can also expect an exponential dependence of tunneling on the center of mass coordinate; the decay of the conduction electron wave function in vacuum is given by the parameter $\lambda$.

Next we introduce the charging (Coulomb) energy of the island with capacitance $C$ (fourth term) and its dependence on the island coordinate (fifth term), written in the form of charge-electric field coupling. The value of $n_{o}$ is controlled by the gate voltage 18. In the present study we will assume that $C$ is small, and $n_{o}$ is very close to $1 / 2$, so that at all relevant temperatures and bias voltages only two charge states, $n=0$ and $n=1$, contribute to the dynamics. We denote the charging energy difference $E_{C}=\left(e^{2} / 2 C\right)\left[\left(1-n_{o}\right)^{2}-n_{o}^{2}\right]$. Since the amplitude of

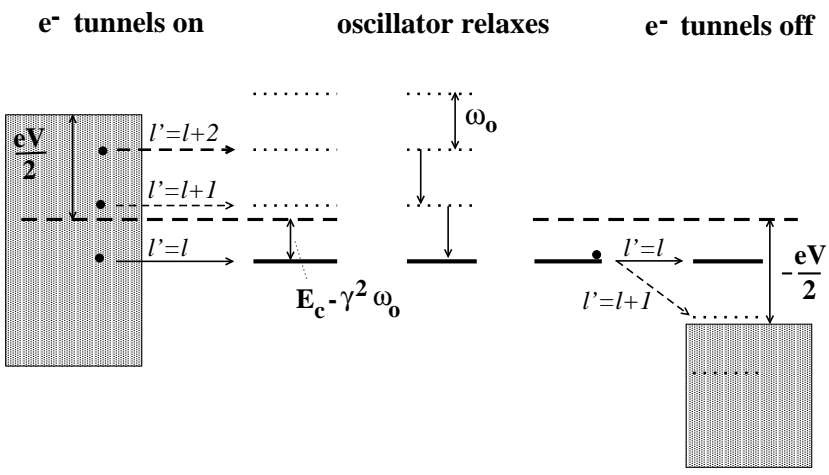

FIG. 1: Schematic of $\mathrm{C}_{60}$ transistor system in terms of combined electronic-vibrational transitions for passage of current through the device. Dashed arrows indicate transitions in which quanta of oscillator energy $\hbar \omega_{0}$ are supplied to the $C_{60}$ by the tunneling electron

zero-point fluctuations, $u_{o}=\sqrt{\hbar / 2 m \omega_{o}}$, is rather small for the heavy island (it is estimated as $\sim 4 \mathrm{pm}$ for the $\mathrm{C}_{60}$ molecule [1]) we do not consider terms non-linear in $x$.

Finally, the last two terms in the Hamiltonian describe an environment and it's dissipative coupling to the molecule via $\hat{V}_{i n t}^{(b a t h)}$. Two mechanisms may contribute here. The vibrational state of the island couples to the bulk and surface vibrations of leads via standard displacement-dependent elastic energy. On another hand, island oscillations produce electrostatic potential fluctuations in the leads which excite electron-hole pairs. We consider the second mechanism as dominant because (i) it is Ohmic dissipative while the phonon coupling is not and vanishes for small energy transfer 15], and (ii) it is mediated by the unscreened Coulomb interaction for the charged island. By writing the coupling term as

$$
\hat{V}_{\text {int }}^{(b a t h)}=\frac{\hat{x}}{u_{o}} \sum_{s k k^{\prime} \sigma} V_{s k k^{\prime}} c_{s k \sigma}^{\dagger} c_{s k^{\prime} \sigma}
$$

we arrive (using golden-rule approximation) at the bathmediated transition rates between vibrational states of the island, $\mid l>$,

$$
\begin{aligned}
W_{l \rightarrow l+1} & =e^{-\hbar \omega_{o} / k_{B} T} W_{l+1 \rightarrow l} \\
& =K(l+1) \frac{\hbar \omega_{o}}{e^{\hbar \omega_{o} / k_{B} T}-1},
\end{aligned}
$$

in the form characterized by a single parameter $K \propto$ $\left(\rho_{F} V\right)^{2}$. [In a more refined theory one may generalize to consider $K$ depending on the island charge as well.] We do not see any obvious reason why this parameter may be anomalously small for leads with metallic concentration of conduction electrons. Thus transitions rates between molecular levels of order $10^{-1} \omega_{o}$ or $10^{-2} \omega_{o}$ must be considered as typical. We performed all our simulations for $K=0.1$. 
In what follows we consider the case of weak conductance when tunneling rates given by $\Gamma^{(0)}=2 \pi \rho_{F} t^{2}$ are much smaller than $K \hbar \omega_{o}$. This condition makes the theory very simple conceptually because one may ignore interference between subsequent tunneling events - on time scales larger than $\hbar / W$ the density matrix of the island reduces to its diagonal form in the oscillator eigenstates representation and one may discuss dynamics in terms of probabilities, $P_{n, \sigma, l}$, of finding a system in a state $|n, \sigma, l\rangle$. The $\mathrm{I}-\mathrm{V}$ curve is then obtained from the steady-state solution of the master equation. This rather standard framework is identical to the one used in Ref. 3. All transition rates for the master equation may be calculated exactly for the model given above. In what follows we explain how this is done and analyze various limiting cases by solving equations numerically. Other work 2] considers the possibility that electronic coherence persists over timescales larger than $\sim 1 / \Gamma^{(o)}$ while assuming undamped classical motion of the oscillator degree of freedom. By contrast, our master equation approach has strong dissipation of the oscillator motion as a built-in condition. In [2] it appears that the stable limit cycles of oscillation are a result of a kinetic stability, and not energetic equilibrium of the oscillator with the environment.

To calculate tunneling matrix elements we have to take care of final-state interaction effects originating from the $\hat{n} E \hat{x}$ term, in other words we first have to diagonalize the Hamiltonian in the absence of tunneling separately for $n=0$ and $n=1$, and calculate matrix elements between the eigenstates corresponding to $n$ values differing by \pm 1 . The resulting $n=0$ and $n=1$ Hamiltonians are related to one another by a shift in space of the potential minimum. Technically, it is convenient to calculate matrix elements in the same basis, and account for the difference between initial and final Hamiltonians by constructing a unitary transformation relating the corresponding eigenfunctions. This is the essence of the Franck-Condon principle. In our case we have to deal with the shifted-oscillator basis set which is a textbook problem. Oscillator states with $n=1$ and $n=0$ are related by

$$
\left|n=1, l>=e^{\hat{S}}\right| n=0, l>
$$

where

$$
\hat{S} \equiv \gamma\left(a^{\dagger}-a\right)
$$

with dimensionless coupling parameter

$$
\gamma=\frac{e E u_{o}}{\hbar \omega_{o}} .
$$

The ground state energy levels are shifted by $-\gamma^{2} \hbar \omega_{0}$.

Since the oscillator Hamiltonian suddenly changes during a tunneling event, the above unitary transformation is all we need in order to calculate matrix elements because it projects $n=1$ states into the $n=0$ Hilbert Space. An effective form of the tunneling Hamiltonian (in the $n=0$ representation) is given now by

$$
\widetilde{H}_{T}=t \sum_{s k \sigma}\left(e^{s \alpha\left(a^{\dagger}+a\right)} e^{\hat{S}} c_{s k \sigma}^{\dagger} c_{i_{\sigma}}+\text { h.c. }\right)
$$

where dimensionless $\alpha=u_{o} / \lambda$ measures the ratio between the molecule zero-point displacement and electron localization length. The energy shift term $-\gamma^{2} \hbar \omega_{o}$ must be taken into account when writing the energy conservation law for all transitions. Specifically, when an island charges/discharges, the tunneling electron will gain/lose an energy equal to the shift. The above considerations are readily generalized for any relevant values of charge states.

So the picture we have is that of the combined electronic-vibrational system with the tunneling Hamiltonian giving dynamics to both degrees of freedom. In the realistic limit of $W \gg \Gamma^{(0)}$, we can assume incoherent dynamics and move to a master equation formulation of the the occupation probabilities of quantum states of the transistor:

$$
\begin{aligned}
\frac{d P_{n, \sigma, l}}{d t}= & -P_{n, \sigma, l} \sum_{n^{\prime}, \sigma^{\prime}, l^{\prime}} \Gamma_{n, \sigma, l \rightarrow n^{\prime}, \sigma^{\prime}, l^{\prime}} \\
& +\sum_{n^{\prime}, \sigma^{\prime}, l^{\prime}} P_{n^{\prime}, \sigma^{\prime}, l^{\prime}} \Gamma_{n^{\prime}, \sigma^{\prime}, l^{\prime} \rightarrow n, \sigma, l} .
\end{aligned}
$$

By considering transitions through only one side of the island (e.g., the right hand side) we obtain the current as

$$
I=e \sum_{\sigma, l, l^{\prime}}\left[P_{1, \sigma, l} \Gamma_{1, \sigma, l \rightarrow 0, l^{\prime}}^{(R)}-P_{0, l}\left(\Gamma_{0, l \rightarrow 1, \sigma, l^{\prime}}^{(R)}\right] .\right.
$$

We have two kinds of transitions in Eq. (10), the thermal rates from (5), which change only the vibrational state $\left(n^{\prime}=n\right)$, and the charge-transfer rates, $\Gamma=\Gamma^{(L)}+\Gamma^{(R)}$, with

$$
\begin{aligned}
& \Gamma_{n, \sigma, l \rightarrow n^{\prime}, \sigma^{\prime}, l^{\prime}}=\frac{2 \pi}{\hbar} \sum_{f i} \rho_{i}^{(e q)}\left|\left\langle f, n^{\prime}, \sigma^{\prime}, l^{\prime}\left|\widetilde{H}_{T}\right| i, n, \sigma, l\right\rangle\right|^{2} \\
& \times \delta\left(E_{f}-E_{i}+\epsilon_{n^{\prime}, \sigma^{\prime}, l^{\prime}}-\epsilon_{n, \sigma, l}\right) ; \quad\left(n^{\prime} \neq n\right), \quad(12)
\end{aligned}
$$

where $n, \sigma$ and $n^{\prime}, \sigma^{\prime}$ are the initial and final electronic state indices for the island, $l, l^{\prime}$ are likewise the initial and final vibrational state indices, and $i, f$ stand for the initial and final states of the leads which are considered to be in the state of thermal equilibrium $\left(\rho_{i}^{(e q)}\right.$ is the equilibrium density matrix of the leads). We also assume the leads to be symmetrically voltage biased, i.e. $+V / 2$ on the left lead and $-V / 2$ on the right one, to avoid unnecessary complications. For the same reason we do not consider the case when tunneling rates $\Gamma^{(0)}$ are different for the left 
and right leads. Explicit expressions for charge-transfer rates are:

$$
\begin{aligned}
\Gamma_{0,0, l \rightarrow 1, \sigma, l^{\prime}}^{(s)} & =\Gamma^{(0)}\left|\boldsymbol{O}_{l^{\prime} l}^{(s)}\right|^{2} f(\delta E) \\
\Gamma_{1, \sigma, l^{\prime} \rightarrow 0,0, l}^{(s)} & =\Gamma^{(0)}\left|\boldsymbol{O}_{l l^{\prime}}^{(s)}\right|^{2}[1-f(\delta E)] \\
\delta E & =E_{c}+\frac{s e V}{2}+\hbar \omega_{o}\left(l^{\prime}-l-\gamma^{2}\right),
\end{aligned}
$$

(where $f(x)$ is the Fermi distribution function) with oscillator matrix elements

$$
\begin{aligned}
\boldsymbol{O}_{l^{\prime} l}^{(s)} & =\boldsymbol{O}_{l l^{\prime}}^{(s)}=e^{-s \alpha \gamma+\frac{1}{2}\left(\alpha^{2}-\gamma^{2}\right)} \sqrt{l^{\prime} ! l !} \\
& \times \sum_{m=0}^{\min \left(l, l^{\prime}\right)} \frac{(s \alpha+\gamma)^{l^{\prime}-m}(s \alpha-\gamma)^{l-m}}{(l-m) !\left(l^{\prime}-m\right) ! m !} .
\end{aligned}
$$

For all simulations we measure energies and voltages in units of $1 \mathrm{meV}$, and current in units of $e \Gamma^{(0)}$.

In calculating IV characteristics from Eqns. (10) and (11), we have to adopt a model for the bias voltage dependence of the electric field $E$ at the position of the island, see (1). Phenomenologically, one might assume there is bias voltage independent component, $E_{0}$, which includes effects of the gate, charged impurities, image charges and screening, device geometry, etc., and bias dependent part proportional to $V / d$ where $d$ is the separation between the leads. Since the electric field for zero and non-zero $\mathrm{V}$ is calculated for the same charge and position of the island, we do not see any physical reason why the dependence of the field on $V$ might be non-analytic. While this effect was implicit in the classical oscillator of [8], it was not considered in [1, 3]. We argue below that it is precisely this term that may help to explain some of the anomalous features in the data of [1] and that negative differential conductance (NDC) may arise in systems in which this terms dominates over $E_{0}$. Indeed, this bias voltage dependence, along with gate voltage dependence of $E_{0}$, represent macroscopic "knobs" with which to control the quantum mechanical transition matrix element between initial and final oscillator states. Thus we have to assume that coupling parameter $\gamma$ is a function of $V$, and write it as

$$
\gamma \equiv \frac{e E u_{o}}{\hbar \omega_{o}} \equiv c_{1}+\left(e V / \hbar \omega_{o}\right) c_{2}
$$

where $c_{1}$ and $c_{2}$ parameterize the relative contributions of bias voltage independent and bias voltage dependent electric fields.

\section{SIMULATION RESULTS}

Since for heavy islands we expect $\alpha \ll 1$ to be a typical situation, we start the analysis from the $\alpha=0$ case first. When voltage dependence of $\gamma$ is ignored (as in Ref. $\underline{3}$ ),

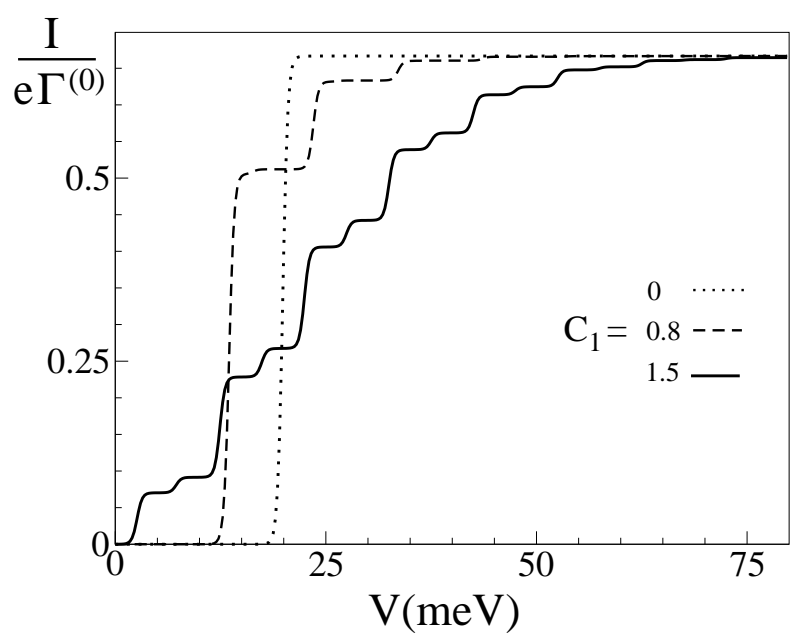

FIG. 2: IV characteristics for $c_{2}=0, c_{1}=0.0,0.8,1.5$ with the voltage offset for each curve defined by the parameter $E_{c}=10 \mathrm{meV}$ (see text)

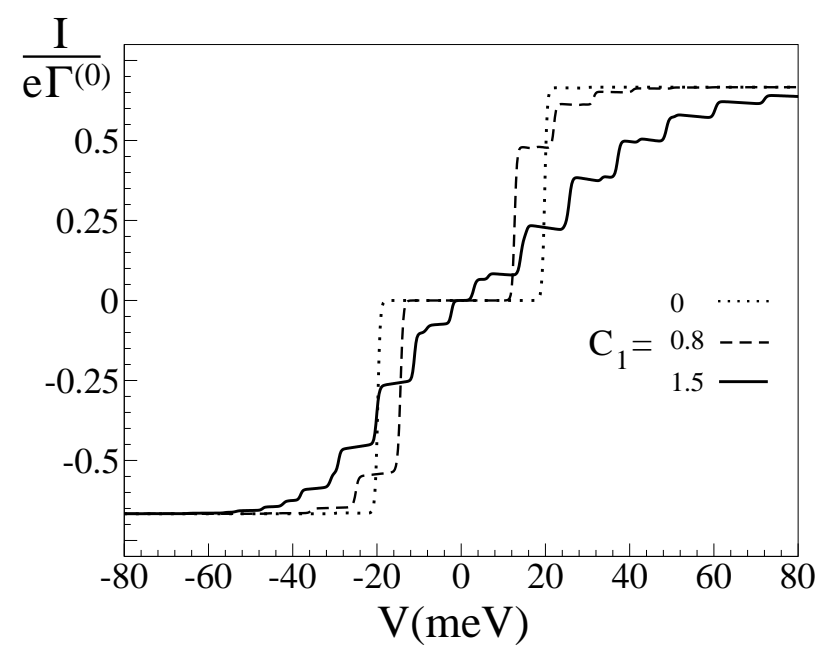

FIG. 3: IV characteristics for finite $c_{2}=0.005, c_{1}=$ $0,0.8,1.5$, and $E_{c}=10 \mathrm{meV}$.

i.e. $c_{2}=0$, we expect that in the limit $V / \hbar \omega_{o}>>\gamma^{2}$ all $\mathrm{I}-\mathrm{V}$ curves must saturate to the ideal value $I_{0}=2 e \Gamma^{(0)} / 3$. The reason for that is in the unitary nature of FranckCondon factors; for zero $\alpha$ we have $\boldsymbol{O}_{l^{\prime} l}^{(s)}=<l^{\prime}\left|e^{S}\right| l>$, and thus when all excitation channels are contributing to the current, we have $\sum_{l^{\prime}}\left|\boldsymbol{O}_{l^{\prime} l}^{(s)}\right|^{2} \equiv 1$. Fig. (2) shows calculated IV characteristics in the strong dissipation limit for $c_{2}=0, c_{1}=0.0,0.3,0.8,1.5$. We have chosen (for all plots) $\hbar \omega_{o}=5 \mathrm{meV}, k_{B} T=0.15 \mathrm{meV}$, to correspond to the experimental data.

There are several observations to be made. First, steps appear at roughly $10 \mathrm{meV}$ intervals, corresponding to $\mathrm{eV} / 2=k \hbar \omega_{o}$, with $k=0,1,2, \ldots$. Note that it is only energy conservation in tandem with non-zero FranckCondon transition matrix elements that gives multiple 


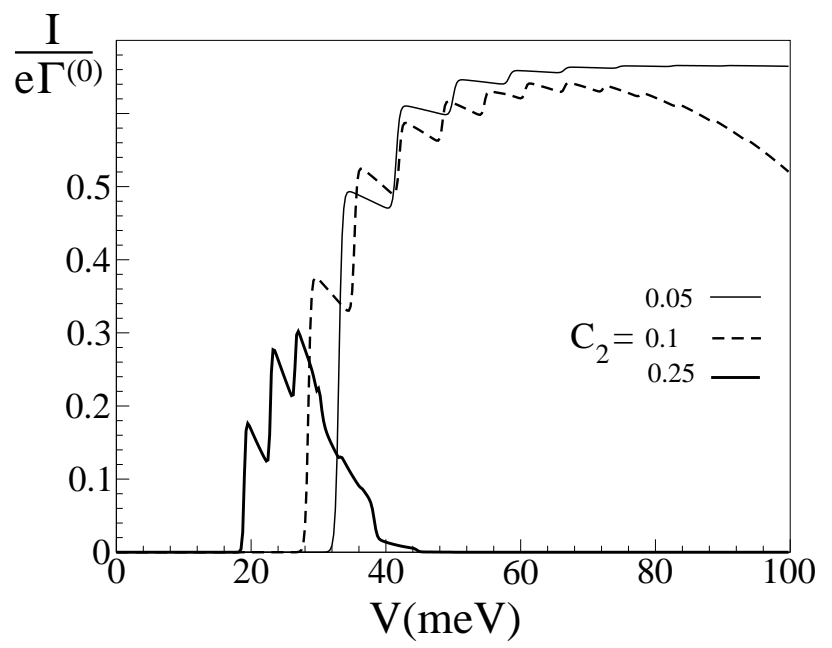

FIG. 4: IV characteristics for $c_{1}=0.5, c 2=0.05,0.1,0.25$, and $E_{c}=20 \mathrm{meV}$

steps. Second, the current through the device saturates to the same limit for all values of $c_{1}$. Also, since the action of the unitary operator is to project the initial oscillator state (in the strong dissipation limit, this initial state is always the ground state of the oscillator) into the eigenbasis of a space-shifted Hamiltonian, we see that the larger the shift parameter $\gamma$, the more access the system has to transitions into states above the ground state of the shifted basis, resulting in more visible steps in the IV characteristic. This is in contrast to the results of Ref. 3 , Fig. 3, where many steps are present when $c_{1}=0$ and the IV curves do not satisfy the unitary limit requirement. The origin of discrepancy is hard to identify since the transition matrix elements were not given explicitly in Ref. 3 .

We note, that for $c_{1}>1$ the structure of the curve depends on the value of the $2\left(E_{c}-\gamma^{2} \hbar \omega_{o}\right) / \hbar \omega_{o}$ parameter because different number of vibrational states are accessible for the electron to enter and to leave the island. These states appear at $2 \hbar \omega$ intervals and form two separate sets which do not coincide if $2\left(E_{c} / \hbar \omega_{o}-\gamma^{2}\right)$ is not integer. When one of the sets reaches its unitary limit before the other set starts conducting (as in the cases $c 1=0.0,0.3$ in Fig. (2) ) we observe only the second set (thus curves with large offset show only one set of steps). For small values of $c_{1}$ conductance is dominated by just one or two states and the second set has no visible effect on the curve ("fades away"). For the largest value of $c_{1}=1.5$ we see that neither set of steps (entering or leaving island) reaches a unitary limit before the other and hence both sets of steps are resolved.

When voltage dependence is accounted for in $\gamma$, Eq. (17), the I-V curves become asymmetric. The obvious effect of non-zero $c_{2}$ is that now the bias voltage affects the unitary transformation. In particular, we note

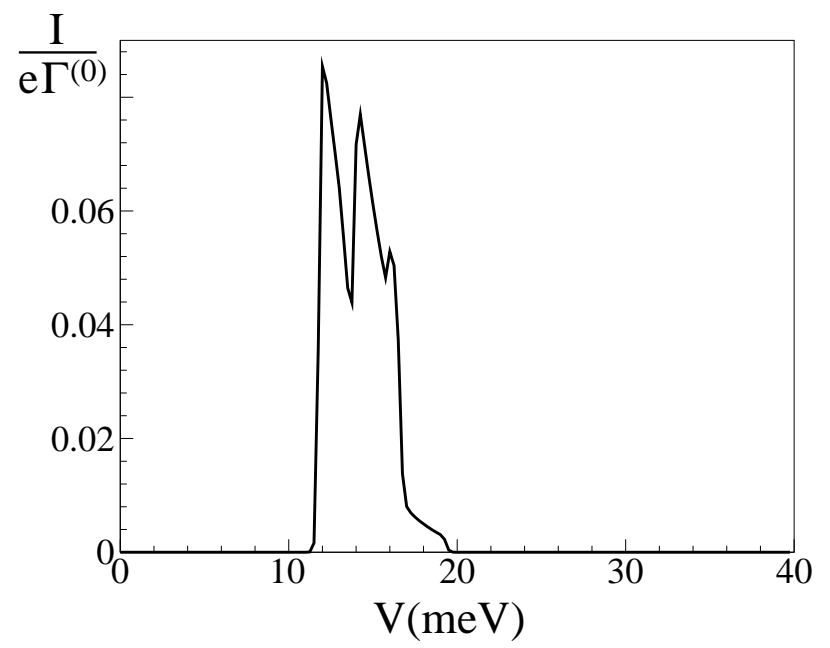

FIG. 5: Conductance resonance due to "catastrophic" negative differential conductance effect for $c_{1}=0.5, c 2=0.5$, and $E_{c}=20 \mathrm{meV}$

negative differential conductance in the intra-step regions on the positive side of bias, while the intra-step regions at negative bias have positive differential conductance, see Fig. (3). This can be explained in the following way: in the intra-step region on the positive side of bias, as voltage increases, the shift parameter $\gamma$ increases, reducing the overlap between the initial oscillator state and the final, shifted state (or states), and hence quantum mechanically constricting that particular $l \rightarrow l^{\prime}$ channel. For negative bias voltages, we note that the constant electric field parametrized by $c_{1}$ and the voltage dependent electric field parametrized by $c_{2}$ work against one another, such that orthogonality between initial and final oscillator states is overall reduced. These I-V characteristics display what appears to be salient in the data of [1], namely an asymmetry about zero voltage in differential conductance, particularly in the intra step regions.

Fig. (4) shows I-V curves for $c_{2}=0.05,0.1,0.25$, with $c_{1}=0.5$ and $\alpha=0$ with charging energy $E_{c}=20 \mathrm{meV}$. Here we see what we refer to as "catastrophic" negative differential conductance. Although the tunneling operator is still unitary, as $c_{2}$ increases, the energy-shift of the final oscillator basis grows strongly (as $\sim-\gamma^{2} \hbar \omega_{o}$ ), with the result that the initial state has its largest projection onto states high in the final-state spectrum. As the bias voltage increases, the orthogonality effect is more and more important, until most of the (oscillator) overlap is in a region where energy cannot be conserved, and so no channels for oscillator transitions remain, giving nearly zero current through the island. Finally, when $\left|E_{c}-\left(c_{1}+\left(V / \hbar \omega_{o}\right) c_{2}\right)^{2} \hbar \omega_{o}\right|$ gets larger than $\mathrm{eV} / 2$ the number of states available for the electron to tunnel off the island is reduced to zero. Fig. (5) shows how this effect can produce a sharp conductance resonance.

Fig. (6) shows the effect of a non-zero $\alpha$. We have set 


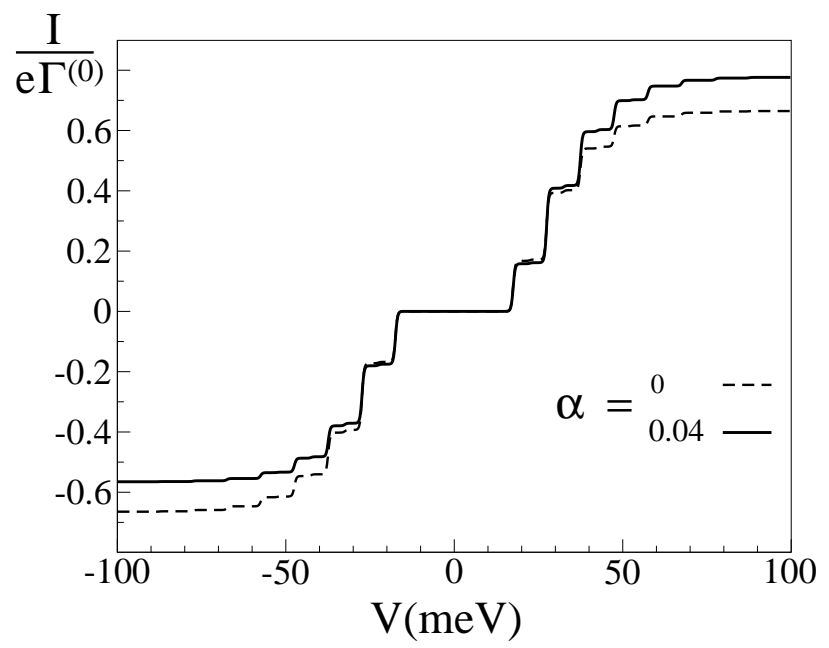

FIG. 6: IV characteristics with $c_{2}=0 ; c_{1}=1.5$ and $\alpha=$ $0,0.04$, and $E_{c}=20 \mathrm{meV}$

$\alpha=0, c 1=1.5$, and $c_{2}=0$ for one of the plots, and $\alpha=0.04, c 1=1.5$, and $c_{2}=0$ for the other. $\alpha=0.04$ is a physically reasonable value which for the $\mathrm{C}_{60}$ case corresponds to the localization length $\lambda \sim 1 A$. Curves with finite $\alpha$ differ only slightly from the $\alpha \rightarrow 0$ case in the magnitude of current at various steps and asymmetry around $V=0$. This is indicative of the manner in which $\alpha$ destroys the unitarity condition $\sum_{l^{\prime}}\left|\boldsymbol{O}_{l^{\prime} l}^{(s)}\right|^{2} \equiv 1$. Since we assume here that zero-charge island states are not shifted, the system conducts better when charged states are shifted towards lower voltage lead. We note that charge transfer physics discussed here radically differs from the semiclassical limit of large-amplitude vibrations presented in Refs. 8 , 2 where electron tunneling was considered to be the fastest process at least for some values of $x$. In our case, finite $\alpha$ does not necessarily mean larger conductance at all voltages. Also, in the semiclassical limit of large-amplitude vibrations the answer crucially depends on dissipation - the vibration amplitude is set by the balance between the energy gained from charge-transfer processes and energy lost to the environment (in the limit of large $c_{1}, c_{2}$ and relatively large $\alpha)$.

\section{CONCLUSION}

Even at the level of the Hamiltonian one can easily see that the dynamics are controlled by many parameters. Although the master equation formally solves the problem for an arbitrary parameter set (as long as dissipation is strong enough), it is difficult to cover all possibilites single paper, even with some of the simplifying assumptions used above. That said, we compare with the $C^{60}$ experiment and find that the IV characteristics can be more or less captured by the $c_{1} \sim 1, c_{2} \sim 0.05$, $\alpha \sim 0.1$ parameter set. It does appear in [1] that there may be features (NDC in the intra-step regions) that reflect the physics of the $c_{2} \neq 0$ case (see Fig. (3)) when the space-shift in the oscillatory potential immediately after a tunneling event depends upon the bias voltage. It is intriguing to note that with $c_{2} \neq 0$ in our model, we have a situation in which the bias voltage, a macroscopically controllable variable, can have an observable influence on the quantum matrix elements which govern the dynamics of the interacting, quantum mechanical degrees of freedom for the system. Future experiments on molecularbased transistors may further explore the existence of such effects, and point the way toward greater control over quantum transitions. The electronically coherent model [2], also seems able to reproduce primary features in the experimental IV curves, but come from otherwise qualitatively different behavior (an classically oscillating island with undamped, stable amplitude $~ 1$ Angstrom, compared with our strongly damped, near zero point motion $(\sim$ picometers $))$. We find that the most interesting physics in our model is the (possibly catastrophic) negative differential conductance effect arising from the experimentally controlled Franck-Condon transition matrix elements.

\section{ACKNOWLEDGMENT}

The authors would like to thank R.V. Krotkov for numerous discussions of shuttling physics.

This work was supported by the National Science Foundation under Grants DMR-0071767 and DMR0071756 .

[1] H. Park, J. Park, A.K.L. Lim, E.H. Andersen, A.P. Alivisatos, and P.L. McEuen, Nature, 407, 57 (2000).

[2] D. Fedorets, L. Y. Gorelik, R. I. Shekhter and M. Jonson, Europhys. Lett., 58, 99 (2002).

[3] D. Boese and H. Schoeller, Europhys. Lett., 54, 668 (2001).

[4] G. M. Whitesides, J. P. Mathias, and C. T. Seto, Science, 47, 254 (1991).

[5] R. P. Andres, T. Bein, M. Dorogi, S. Feng, J.I. Henderson, C.P. Kubiak, W. Mahoney, R.G. Osifchin, and R. Reifenberger, Science, 47, 272 (1996).

[6] D.I. Gittins, D. Bethell, D.J. Schiffrin, and R.J. Nichols, Nature, 47, 408 (2000).

[7] Y. Eichen, E. Braun, U. Sivan, and G. Ben-Yoseph, Acta Polym., 47, 49 (1998).

[8] L.Y. Gorelik, A. Isaacson, M.V. Voinova, B. Kasemo, R.I. Shekhter, and M. Jonson, Phys. Rev. Lett., 47, 80 (1998).

[9] M.T. Tuominen, R.V. Krotkov, and M.L. Breuer, Phys. Rev. Lett., 47, 83 (1999). 
[10] A. Erbe, C. Weiss, W. Zwerger, and R.H. Blick, Phys. Rev. Lett., 47, 87 (2001).

[11] S.H.M. Persson, L. Olofsson, and L. Gunnarson, Appl. Phys. Lett., 47, 74 (1999).

[12] E.S. Soldatov, V.V. Khanin, A.S. Trifonov, D.E. Presnov, S.A. Yakovenko, G.B. Khomutov, C.P. Gubin, and V.V. Kolesov, JETP Lett., 47, 64 (1996).

[13] J.R. Petta, D.G. Salinas, and D.C. Ralph, Appl. Phys.Lett., 47, 77 (2000).

[14] H. Park, A.K.L. Lim, A.P. Alivisatos, J. Park, and P.L. McEuen, Appl. Phys. Lett., 75, 2 (1999).

[15] A.J. Leggett, S. Chakravarty, A.T. Dorsey, M.P.A.
Fisher, A. Garg, and W. Zwerger, Rev. Mod. Phys. 59, 1 (1987).

[16] L.Y. Gorelik, A. Isaacson, Y.M. Galperin, R.I. Shekhter, and M. Jonson, Nature, 47, 411 (2001).

[17] L. Clark, M. N. Wybourne, L. O. Brown, J.E. Hutchison, M. Yan, S.X. Cali, and J.F.W. Keana, Semiconductor Sci. and Technol., 47, 13 (1998).

[18] H. Grabert and M.H. Devoret (Eds.), Single Charge Tunneling: Coulomb Blockade in Nanostructures, Plenum Press, New York-London, (1992). 